\title{
USE OF A MOBILE ARCHITECTURAL GUIDE
}

\author{
ALEXANDER KOUTAMANIS \\ Delft University of Technology, The Netherlands
}

\begin{abstract}
A mobile architectural guide assists architectural tourists in selecting, navigating to and recognizing architectural sights. The guide supports such tasks by means of domain knowledge, design documentation and contextual information organized into navigation modules, architectural information systems and learning modules.
\end{abstract}

\section{Visiting Architecture}

Modern information and communication technologies support access to all kinds of information from virtually any place in several continents. Access to online information is no longer restricted to conventional computing devices: mobile telephones and other lightweight wireless devices are becoming increasingly popular for information processing on the road or in the field. Despite the obvious limitations of screen size, processing and storage capacity, such devices are generally capable of supporting interaction with the majority of documents used in professional settings. The current focus in mobile information processing seems firmly fixed on 'social' applications but there is a discernible increase in more conventional professional applications (Kaga et al. 2006; Matsumoto et al. 2001; Tedrumpun and Nakapan 2004; Wang and Shih 2002) - even if it may have come rather late, as we can judge from the recent stagnation in the development of palmtop hardware and software. Somewhere in between social and professional applications we encounter informative and educational applications which make use of professional information for non-professional purposes and use technologies marketed for personal entertainment and communication.

Digital architectural guides are an example of such applications. Even though copyright issues form obstacles of increasing magnitude and complexity, there has been substantial even though unsystematic digitization of information on architecturally important buildings. Online resources concentrate mostly on visual documentation (scanned drawings, photographs, three-dimensional models), while only a few have been 
designed as architectural guides, with a complete presentation of a building and its context in mind (including maps and instructions on how to get there, e.g. www.galinsky.com). Nevertheless, with the plethora of navigation services and maps on the Internet the addition of visiting information to databases of buildings has become quite straightforward (e.g. www.greatbuildings.com).

An architectural guide must fulfill a number of requirements. It should provide concise but unambiguous information that facilitates identification and a fundamental understanding of a building. It should be legible, lightweight and comprehensive. It should support searches and browsing from various points of view and entry points, e.g. location, style, architect, type etc. All these functions and constraints relate to the activities of visiting one or more buildings either casually (as part of various tourist attractions) or specifically (as architectural tourist). Paper guides remain a favorite among architectural tourists but are often criticized for the quality of their visual documentation (small size, vagueness, incompleteness or lack of photographs and drawings), for their coverage (i.e. being overly selective, arbitrary or not up-to-date) and the precision and accuracy of contextual information (especially the part that supports finding a building). Most complaints derive from the analogue nature of publications on paper and the low periodicity of architectural guides (Koutamanis 1998). It is therefore surprising that there has been so little attention for digital guides, especially on mobile devices which can be carried around just like their paper counterparts.

In recent years there has been some interest in architectural guides on mobile devices but mostly from mobile telephony providers or related service providers (cultural or tourist). It is already possible to make architectural walks with one's own mobile phone providing textual or aural instructions and commentary in several cities (www.talktomenl.nl, www.walkthetalk.hk). In CAAD research there is only one known relevant research project, thankfully with extensive ambitions and many facets (Berridge and Brown 2002; Berridge et al. 2002; Brown et al. 2006). The wide general interest in technologically related subjects like ubiquitous computing and smart buildings has returned interesting ideas on e.g. interaction with information and navigation, especially in confined environments like museums and historical areas (Bruns et al. 2005; Jeng 2005; Nomura and Kishimoto 2005, Shen and Teng 2005), but no architectural guides as primary products. Interest in guided navigation with information feedback has been higher in other fields, with emphasis mostly on general computational and cognitive issues (Abowd et al. 1997; Cheverst et al. 2000; Persson et al. 2002). 
In addition to their educational value, digital architectural guides present new research opportunities: by making already available digital information accessible for mobile use we not only extend the utility of digital documentation and explore the potential of mobile information processing (Berridge et al. 2003) but also investigate the wider usability and applicability of existing representations and information systems towards more effective and efficient fundamental solutions to information and modeling problems (Knight et al. 2006). The two questions of how to make existing information available for mobile processing and what information is necessary for mobile architectural applications are central to our research into the development of a mobile architectural guide.

\section{A Mobile Architectural Guide (MAG)}

MAG is a modular system that has been implemented on a number of mobile platforms (palmtop computers and smartphones with various operating systems). In general lines MAG consists of:

1. Architectural information systems containing multimedia descriptions of the sights: texts, drawings, photographs, video etc. that present a building, its context and background to an architectural public.

2. Navigation modules that permit location identification, support orientation and assist organizing a trip to a number of sights.

3. Learning modules that register, remember and re-use user preferences and search patterns.

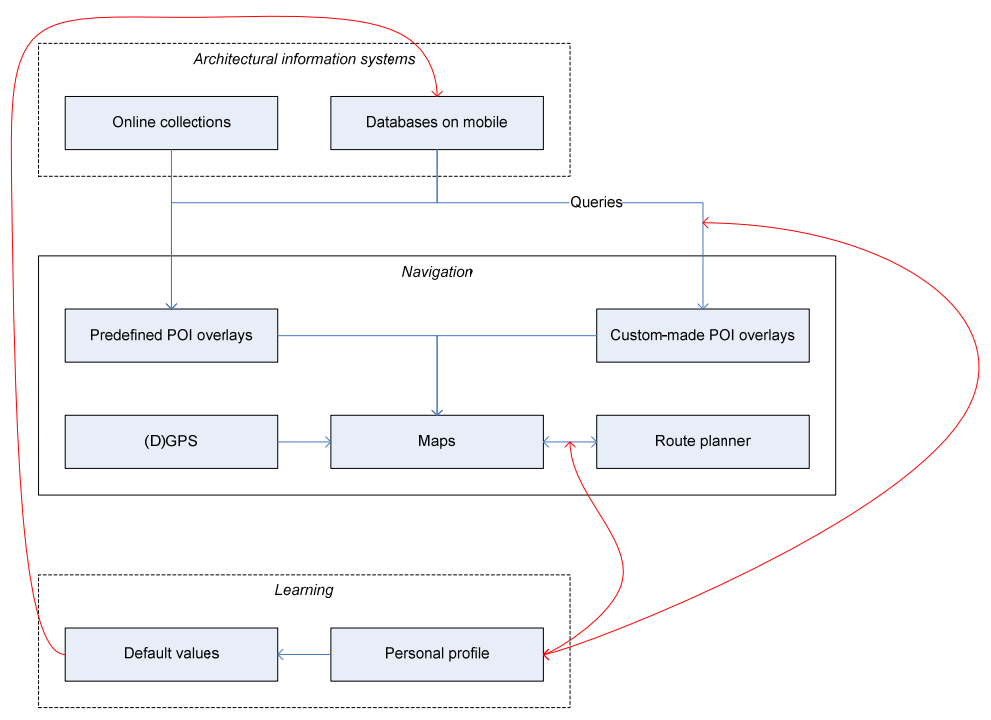

Figure 1. MAG outline 
The main intention concerning MAG use is to assist architectural tourists in identifying and navigating to architectural sights. Such information could be also made available to other tourists with a casual cultural interest in architecture but the demands of the architectural visitor are significantly higher and result into more challenging specifications and problems.

The architectural information systems of MAG can be purpose-made databases on a mobile device as well as external online collections, which are connected to the MAG either directly (e.g. as a hyperlink to a web page) or through a linking database on the mobile device. The connection mode depends on the structure of the external collection, i.e. support for direct retrieval of relevant items (e.g. by having a standardized content subdivision into separate web pages). Problems relating to the size of items to be retrieved have been left outside the scope of the first implementations of MAG.

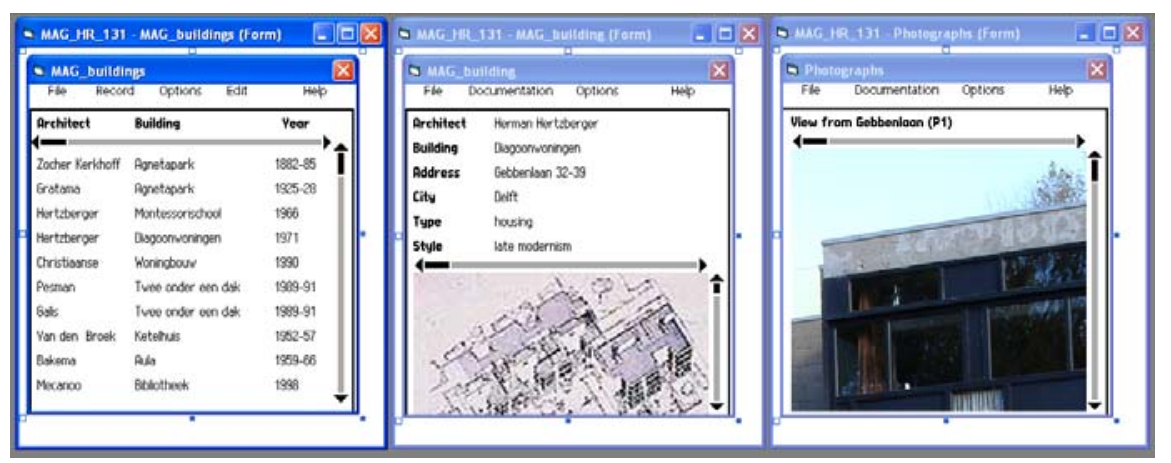

Figure 2. Mobile architectural database in MAG

The navigation modules are built on top of existing navigation systems that provide the general functionality a tourist requires. With respect to navigation there are three main options: (i) smart tagging of architectural sights, (ii) use of mobile phone network cells, or (iii) use of mobile navigation systems based on satellite global positioning (GPS) or combined satellite-terrestrial differential global positioning (DGPS). We have chosen for the third option because it needs no dedicated infrastructure, offers greater accuracy, precision and sensitivity, and links seamlessly architectural interests with general tourist support (Koutamanis. 2006). This also means that users can add a MAG to their own navigation system and reduce learning time and cost. The main disadvantage of dependence on external commercial R\&D is alleviated by linking navigation systems with MAG by means of universal overlays of points of interest (POI). Such overlays can be used with practically any navigation system and form a logical extension of the architectural databases in MAG (i.e. a summary of pragmatic 
information: textual and geographic data for identifying a building and its location).

The learning modules facilitate interaction with the MAG by recording and re-using user input and interaction with different modules. Consequently, the learning part of MAG forms an interconnected network of modules distributed in overlay facilities (e.g. where users enter their own POI), the navigation system (e.g. registration of transport mode preferences and success rate in actually visiting the scheduled sights), and especially in architectural information systems, where users can not only add new sights but also define selection criteria that reflect their individual interests in particular architectural styles, periods, architects, building types etc. Our interest in learning lies mainly in its important for the efficiency and effectiveness of user interaction with MAG - two primary concerns in mobile information processing. Being able to anticipate user actions reduces response time and redundancy in the options offered at any given moment without diminishing the flexibility and comprehensiveness of the overall system.

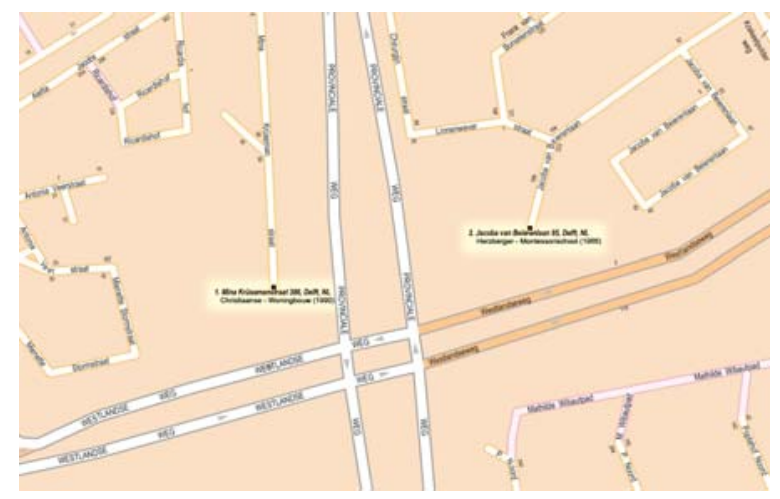

Figure 3. MAG architectural sights overlay in a navigation system map (detail)

\section{Navigation: Routing}

The focus of the present paper is primarily on the navigation modules and secondarily on the architectural information systems in their capacity of information sources that support orientation and recognition. This combination supports both local and global navigation capabilities, i.e. respectively enables effective and reliable interaction with the built environment (including understanding of one's own motion, recognition and avoidance of obstacles, and achieving a stable mental image of the environment) and facilitates the development of mnemonic structures with reference to the environment (Fermüller and Aloimonos 1995). In the case of the MAG we are interested in the first (local navigation capabilities) as a 
practical necessity and in the second (global capabilities) as the ultimate goal of an architectural navigation and information aid.

In a typical MAG implementation navigation relies on a commercial satellite navigation system in a palmtop computer or smartphone. Buildings in the architectural information system are imported to the navigation system as POI overlays. Each POI serves not only as identification of the building and its location but also as a dynamic link to the architectural information system, so that users can move seamlessly between the navigation and the information systems. The number of architectural overlays depends on a small number of criteria, primarily the number of architectural sights in a guide and the diversity of building styles and types in the information system. Users of databases on the mobile device can define their own POI overlay distribution on the basis of queries and reports in the database which can generate new overlay files. Such actions have an impact on the system's learning, as the queries and overlays are included in the particular user's profile.

Navigation operates in two different modes. The first is based on the identification of intervening opportunities and forms a straightforward application of capabilities existing in many commercial systems: the navigation system alerts the user to the existence of an architectural sight in the area on the basis of dynamic proximity (i.e. taking into account the direction and speed of movement). Users have then the option of adapting their current route so as to visit the building. The second mode is planned visits and tours. In this mode users start either in the navigation or the architectural information system with a selection of buildings and organize a route that allows them to visit every building in the selection. The route can be the shortest one, thematic, chronological or any other order supported by the architectural information system and can utilize any transportation means supported by the navigation system.

Combining such criteria for ordering architectural sights in a reasonable route is a complex process which may be poorly supported by route planners in commercial navigation systems. To compensate for eventual limitations and integrate the required domain knowledge we have added a sorting module that organizes the results of a query on the basis of multi-criteria techniques. The purpose of this module is to allow the user to consider his priorities and minimize unnecessary complexity in the route. The latter is performed by means of a preliminary evaluation of the routes proposed by the navigation system on the basis of a topological analysis we have previously applied to pedestrian circulation in buildings (Koutamanis et al. 2001). This evaluation reduces self-intersections in route and draws the user's attention to lengthy links between nodes in the route. 
One of the reasons why such preprocessing and evaluation is necessary is the difference between proximity and accessibility: architectural sights may be next to each other and yet separated by major obstacles such as a railway line or a waterway that do not afford crossing in the immediate vicinity. The MAG is particularly sensitive to such problems that might spoil architectural touring with irrelevant time-consuming and cumbersome activities. Preprocessing of routes to architectural sights is more extensive when the complexity of their physical context makes navigation and identification of the route too cumbersome. In such cases the building has an own local navigation overlay that presents a number of options and connections, e.g. pedestrian footpaths leading to the building from the nearest public transportation stops or a parking lot (Figure 5).

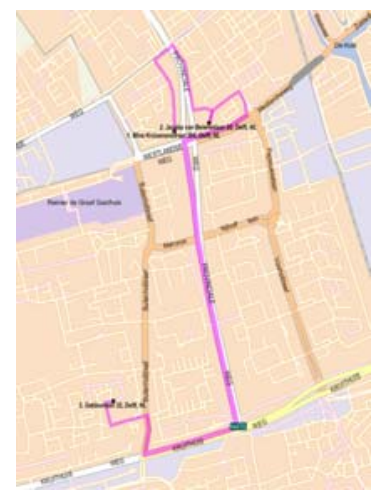

Figure 4. MAG routes: corrected route linking three sights by car

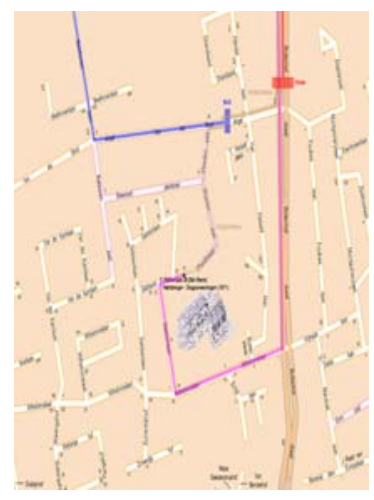

Figure 5. MAG routes: local navigation options focused on a single sight

Connecting local navigation options to the mental maps made by tourists and (in the terminology of Lynch) place legibility is questionable. It could be argued that these options and interaction with routes proposed by a navigation system in general antagonizes the paths tourists perceive. It is not 
uncommon that users of navigation systems have difficulty mapping the visual representations of a route on a map to the scenes they perceive. The pseudo-three-dimensional projections currently in vogue do little to reduce this difficulty. It could be further argued that navigation aids should focus more on landmarks, edges, nodes and other places where visual and other cues combine to assist place legibility and the formation of reference frameworks. Such important questions are left out of the scope of the first implementations of MAG, which is restricted to more practical navigation issues. Local navigation support is therefore adapted to the strengths and possibilities of the host navigation systems. One notable exception that may provide a glimpse of a more coherent and effective investigation of place legibility is orientation support. The reasons for providing such support remain nevertheless practical.

\section{Navigation: Orientation}

Even when routing problems are satisfactorily solved, the architectural tourist may still experience difficulty reaching a building as a result of orientation problems. In an unknown environment one often has difficulty identifying the right direction, especially under inconvenient conditions (e.g. cloudy skies, insufficient or confusing road signboards, obscure paths). Orientation problems are compounded by the inability of (D)GPS-based navigation systems to identify directions or even the current location when the user is stationary or moving slowly. The resulting uncertainty may lead to frustration and distrust of the navigation system and the MAG.

Our approach is to provide information that supports effective, efficient and reliable orientation. Such information consists of local overlays that focus on the accessibility of a building (Figure 5) and visual descriptions of the context at critical points (nodes) in a route (crossroads, public transportation stops, parking lots), such as photographs of easily recognizable landmarks taken from these points and panoramic views. These photographs are annotated with indications of the cardinal directions and the road leading to the target of the route or other significant points (Figure 6).

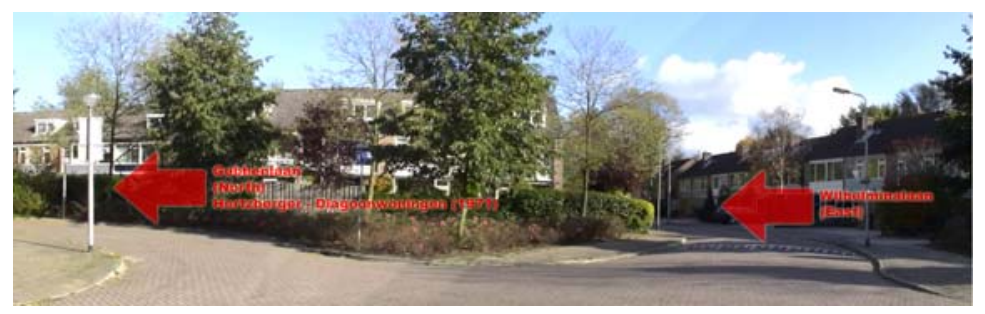

Figure 6. Orientation panorama with navigation instructions 
An alternative to static panoramas are short panoramic videos (pan shots), which can also indicate movement towards the next target (traveling or zoom shots of a building, landmark, crossing etc.). Such videos can be surprisingly small in storage size, efficient in use and quite legible on the small screens of mobile devices, frequently performing better than static panoramas in terms of recognition.

\section{The MAG in Use}

Experimental use of the first versions of the MAG focused on a few small geographic areas containing 12-36 architectural sights. The developers of the system were familiar with some of these areas and first-time visitors to others. This combination allowed for evaluation on the basis of established experiential expectations as well as general functional specifications. The navigation and information support provided by the MAG was appreciated by users in all cases, as it supported a higher precision in recognizing a building and its environment than analogue guide books. Such support was particularly useful in cases where precise identification of an architectural sight was made rather tentative by later buildings in the immediate area that imitated the morphology of the eponymous ones. The MAG assisted in the resolution of such problems by providing not only precise navigation aids but also more extensive information than guide books.

The routing aids included in the MAG were useful in two respects. The first was the ability to make selections of buildings either in the navigation or the architectural information system. The existence of multiple entry points allowed users to interact flexibly with the MAG, even extensively adapting routes on the way. The domain knowledge in the architectural information systems was intensively used, as it supported transparent selection on the basis of different criteria. The second was avoidance of topographical features that spatially separated a selection of buildings into two or more subgroups. Elevated railway lines and rivers were probably the hardest obstacles because the existence of respectively passageways on ground level and bridges meant that unsupported route planners could suggest routes that seemed logical because they did not contain long links. On the other hand, users have commented that by attempting to make routes more efficient one may sacrifice the coherence or consistency of a thematic architectural tour. Such choices are quite transparent in the queries but user interaction with a mobile device appears to afford too little time for reflection and elaboration. Most users tended to avoid cyclical routing processes.

One of the critical observations made during early use of the MAG is that architectural images may be removed from normal everyday perception of the built environment: they often relate strongly to canonical representations 
and views which explain aspects of design rather than present the appearance of a building from trivial viewpoints that may nevertheless assist recognition. The inclusion of photographs and video in the documentation may resolve most related problems of the architectural tourist but at a cost: increase in the redundancy of information in the MAG requires more storage space and relatively high processing capacity (especially for efficient transitions between modules and programs). Arguably more significant is that, as architectural tourists also want to understand and analyze the buildings they visit, users are directly confronted with this redundancy. Multimedia techniques can make relationships between information items transparent but cannot resolve problems of inconsistency, incompleteness and overload.

The ability to add as much information as possible (available) to a digital information system does not mean that the system becomes more informative. While we firmly believe than practically all users can use their cognitive faculties to discriminate between relevant and irrelevant, crucial and trivial information on the basis of variable personal criteria, we do not want to succumb to the temptation of developing an all-encompassing documentation system for architectural monuments. We do not have the illusion that a user can learn a building from a guide book (even if digital) and a visit. Consequently we restrict the applicability of the MAG to support for finding and recognizing the building, and to explaining salient aspects and interesting features like construction details and development stages that might become apparent during a visit. This also means that we had to resist suggestions to include more information on the context of buildings (e.g. extensive historical reviews of city parts).

A second critical user observation is that current navigation systems contain incomplete cartographic data for cyclists and pedestrians. As a result of the mapping systems used for the collection of such data, cycle and pedestrian routes may be adequately documented only if they are close to motorways. This has consequences for the clarity and efficiency of the last parts of a route (near a building). The MAG compensates for such limitations by means of additional local information (Figure 5). These improve the effectiveness and reliability of the system without significant loss of efficiency, even on less powerful mobile devices (especially if added to the navigation systems as overlays).

\section{Discussion}

From a technical viewpoint the MAG is essentially similar to multimedia systems we have been developing for different purposes and platforms since the mid-1990s. Current mobile devices present few technical problems to the development of such systems, even with demanding types of information 
like three-dimensional models and video. The usual complaint of small screen sizes presented few obstacles to the design and development of the various MAG versions because the spatial resolution of current devices is generally sufficient for the visual information we need in a digital guide for architectural tourists.

The extensive use of existing digital building documentation in the MAG caused few technical problems but also made evident essential differences between design information and the perception of real buildings. These differences range from the variable abstraction used in architectural projections (which makes many drawing scales inappropriate as conveyors of spatial information) to that most viewpoints used in architectural projections are far removed from those where a visitor can actually stand. Such differences point to the fundamental dichotomy of technical and presentation aspects in architectural representation.

In subsequent versions of the MAG we intend to address such issues by focusing more on three-dimensional models that integrate different projections and perspective transformations of two-dimensional images so as to match visitors' viewpoints. Such representations also have a more direct relationship with advanced Euclidean and projective representations proposed in visual navigation studies (Riseman et al. 1997; Robert et al. 1997), as well as with mental spatiotemporal representations of visual memories (Aloimonos et al. 1995; Nelson 1997). Another area of improvement concerns the application of automated visual recognition to the recognition of objects. Recent advances suggest that mobile devices could accommodate feature-based systems that can distinguish between members of one or more classes (Föckler et al. 2005). Resolution issues may impede recognition of a building as a whole but identification of particular or typical elements and salient features such as classical columns of a specific order seems to be feasible also for MAG. Recognition of such features presents new opportunities for learning (machine and human) as well as new possibilities for search entries.

Another extension that was actually suggested by an anonymous reviewer of this conference is the use of MAG to study mental maps and place legibility. The purpose of this is twofold: on the one hand the improvement of local navigation and orientation without resorting to predetermined paths and on the other the refinement of our understanding of architecture in context. The first seems feasible despite the limitations of mobile devices and host navigation systems but the latter may require significant technological additions for registering and analyzing human behavior. In the resulting system MAG will probably play a secondary role.

The main operational problem of MAG remains maintenance, in particular of the contextual information that contributes to identification, 
orientation and recognition. Given the growing popular interest in navigation systems, we expect that we can rely on their developers for up-to-date cartographic data and improvements in route planning. However, we also expect that our own additions will remain at least partially useful and that they will require similar periodic verification and improvement.

\section{References}

Abowd, GD, Atkeson, CG, Hong, J, Long, S, Kooper, R and Pinkerton, M: 1997, Cyberguide: A mobile context-aware tour guide, Wireless Networks 3(5): 421-433.

Aloimonos, Y, Fermüller, C and Rosenfeld, A: 1995, Seeing and understanding: representing the visual world, ACM Computing Surveys 27(3): 307-309.

Berridge, $\mathrm{P}$ and Brown, A: 2002, A touring machine, Connecting the Real and the Virtual design e-ducation. 20th eCAADe Conference Proceedings, eCAADe, Warsaw, pp. 488493.

Berridge, P, Brown, A and Knight, M: 2002, One city to go, in AME Rafi, CW Khong, M Neo, KT Neo and SNAS Ahmad (eds) CAADRIA 2002. Proceedings of the 7 th Conference on CAAD Research in Asia, CAADRIA, Cyberjaya, pp. 57-64.

Berridge, P, Koch, V and Brown, AGP: 2003, Information spaces for mobile city access, International Journal of Architectural Computing 1(1): 34-45.

Brown, A, Knight, M, Chen, Y-H and Saeed, G: 2006, City information delivered to mobile digital devices - reflection on contemporary potentail and problems, in $\mathrm{V}$ Bourdakis and $\mathrm{D}$ Charitos (eds) Communicating space(s). eCAADe, Volos, pp. 146-150.

Bruns, E, Brombach, B, Zeidler, T and Bimber, O: 2005, Enabling mobile phones to support large-scale museum guidance, Bauhaus University Weimar.

Cheverst, K, Davies, N, Mitchell, K, Friday, A and Efstratiu, C: 2000, Developing a contextaware electronic tourist guide: some issues and experiences, Proceedings of the CHI'00. ACM international conference on human factors in computing systems, ACM Press, New York, pp. 17-24.

Fermüller, C and Aloimonos, Y: 1995, Vision and action, Image and Vision Computing, 13(10): 725-744.

Föckler, P, Zeidler, T, Brombach, B, Bruns, E and Bimber, O: 2005, PhoneGuide: museum guidance supported by on-device object recognition on mobile phones, International Conference on Mobile and Ubiquitous computing (MUM'05). ACM Press, New York, pp. 3-10.

Jeng, T: 2005, Advanced ubiquitous media for interactive space, in B Martens and A Brown (eds) CAAD Futures 2005, Kluwer, Dordrecht, pp. 341-350.

Kaga, A, Miyagawa, A, Kawaguchi, M, Yeo, W and Fukuda, T: 2006, Landscape evaluation system using a 3D space model and a cellular phone with GPS camera, in A Kaga and R Naka (eds) CAADRIA 06. Proceedings of the 11th Conference on CAAD Research in Asia, CAADRIA, Kumamoto, pp. 503-512.

Knight, MW, Brown, AGP and Smith, JS: 2006, Digital terrain meshes from GPS in urban areas: A practical aid to city modelling, in A Kaga and R Naka (eds) CAADRIA 06, Kumamoto, pp. 443-451.

Koutamanis, A: 1998, Information systems and the Internet: towards a news counterrevolution?, 4th Design and Decision Support Systems in Architecture and Urban Planning Conference, Eindhoven.

Koutamanis, A: 2006, Contextual awareness in mobile information processing, in $\mathrm{V}$ Bourdakis and D Charitos (eds) Communicating space(s), eCAADe, Volos, pp. 152-159. 
Koutamanis, A, van Leusen, M and Mitossi, V: 2001, Route analysis in complex buildings, in B de Vries, J van Leeuwen and H Achten (eds) CAADFutures 2001, Kluwer, Dordrecht, pp. 423-430.

Matsumoto, Y, Onishi, Y, Yamaguchi, S and Morozumi, M: 2001, Using mobile phones for accelerating interaction, in $\mathrm{H}$ Penttilä (ed) Architectural information management, eCAADe \& Helsinki University of Technology, Espoo, pp. 311-316.

Nelson, RC: 1997, From visual homing to object recognition, in Y Aloimonos (ed) Visual navigation, Lawrence Erlbaum Associates, Mahwah, New Jersey, pp. 218-250.

Nomura, Y and Kishimoto, T: 2005, Visualization of tourists' behavior and activity using GPS and GIS in Kamakura City, in A Bhatt (ed) CAADRIA 2005, Seoul, pp. 320-327.

Persson, P, Espinoza, F, Fagerberg, P, Sandin, A and Cöster, R: 2002, GeoNotes: A locationbased information system for public spaces, in K Höök, D Benyon and A Munro (eds) Designing information spaces: The social navigation approach, Springer, London.

Riseman, EM, Hanson, AR, Beveridge, JR, Kumar, R and Sawhney, H: 1997, Landmarkbased navigation and the acquisition of environmental models, in $\mathrm{Y}$ Aloimonos (ed) Visual navigation, Lawrence Erlbaum Associates, Mahwah, New Jersey, pp. 317-374.

Robert, L, Zeller, C, Faugeras, O and Hébert, M: 1997, Applications of nonmetric vision to some visually guided robotic tasks, in Y Aloimonos (ed) Visual navigation, Lawrence Erlbaum Associates, Mahwah, New Jersey, pp. 89-134.

Shen, Y-T and Teng, T-S: 2005, Personal mobile device for situated interaction, in A Bhatt (ed) CAADRIA 2005, Seoul, pp. 382-387.

Tedrumpun, T and Nakapan, W: 2004, Site measurement with combination of pocket PC and global positioning system for preliminary architectural design process, in J-W Choi and HS Lee (eds) CAADRIA 2004, Seoul, pp. 717-726.

Wang, P-H and Shih, N-J: 2002, A preliminary application of a PDA-based OODB system for construction information management, in K Kosszewski and S Wrona (eds) Connecting the real and the virtual - design e-ducation, eCAADe, Warsaw, pp. 306-309. 